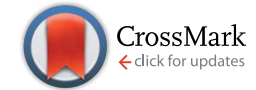

Cite this: RSC Adv., 2017, 7, 4776

Received 29th November 2016 Accepted 31st December 2016

DOI: $10.1039 / c 6 r a 27480 c$

www.rsc.org/advances

\section{Brush-like block copolymer synthesized via RAFT polymerization for graphene oxide aqueous suspensions}

\begin{abstract}
Min Qiao, ${ }^{\text {ab }}$ Shishan Wu, ${ }^{\text {*a }}$ Yanwei Wang ${ }^{b}$ and Qianping Ran ${ }^{b}$
A brush-like block copolymer of poly(acrylic acid)-block-poly(oligo(ethylene glycol)methyl ether acrylate) (PAA-b-POEGA) was synthesized via reversible addition-fragmentation chain transfer (RAFT) polymerization, and its dispersing ability as polymeric dispersant for graphene oxide (GO) sheets in aqueous suspensions was studied in comparison with a traditional random copolymer having similar chemical units. The graphene oxide aqueous suspensions remained stable at a GO concentration as high as $100 \mathrm{~g} \mathrm{~L}^{-1}$ for more than 7 days with the assistance of the prepared brush-like block copolymer, which could not be fulfilled by the random copolymer control group as dispersant. Measurements on polymer adsorption, zeta potential, shear viscosity, particle size analysis, and by small angle X-ray diffraction and scanning electron microscope were carried out to characterize the improved dispersing ability of the brush-like block copolymer for GO aqueous suspensions.
\end{abstract}

\section{Introduction}

Graphene, a two-dimensional sheet of single atomic thick layer of hexagonally arranged carbon atoms,${ }^{1,2}$ has attracted significant interest due to its extraordinary physical properties which make it an ideal candidate for a wide range of applications, such as actuators, ${ }^{3,4}$ super capacitors, ${ }^{5}$ hydrogen storage, ${ }^{6}$ nanocomposites. ${ }^{7,8}$ Graphene oxide (GO), which is obtained via the oxidative exfoliation of natural graphite, can be used as a solution-processable precursor for the bulk production of graphene. ${ }^{9}$ Although the dispersion of GO in aqueous solution is easier than graphene due to its hydrophilic surface groups, the aggregation of GO caused by van der Waals interactions may still restrict its applications. ${ }^{10}$ Several stabilizers, such as surfactants, ${ }^{11,12}$ polymeric dispersants, ${ }^{13-17}$ and aromatic or $\pi-\pi$ stacking molecules, ${ }^{18}$ can be applied to prevent the aggregation of GO sheets. Among the above methods, polymeric dispersants are considered as the most effective approach to prepare the highly stable graphene aqueous suspensions. In recent years, block copolymers are found to have good dispersing abilities for $\mathrm{GO}$ in aqueous suspensions. Yan et al. ${ }^{19}$ employed the pluronictype block copolymers to get stable GO aqueous suspensions. Hyang et al. ${ }^{20}$ synthesized an amphiphilic fluorinated block copolymer via reversible addition-fragmentation chain transfer (RAFT) polymerization for the aqueous dispersion of GO. The

${ }^{a}$ School of Chemistry and Chemical Engineering, Nanjing University, Xianlin Campus, 163 Xianlin Avenue, Qixia District, Nanjing 210023, P. R. China. E-mail: shishanwu@ nju.edu.cn

${ }^{b}$ State Key Laboratory of High Performance Civil Engineering Materials, Jiangsu Sobute New Material Co. Ltd, 118 Liquan Road, Jiangning District, Nanjing, 211103, China amphiphilic fluorinated block copolymer can effectively prevent aggregation of GO over a period of 7 days at a GO concentration of $50 \mathrm{~g} \mathrm{~L}^{-1}$. Perumal et al. ${ }^{21}$ used a linear amphiphilic copolymer of poly(4-vinyl pyridine)-block-poly(ethylene oxide) (PVP-b-PEO) to get stable aqueous and ethanolic GO dispersions, and the prepared polymer dispersant was able to prevent the aggregation of GO in aqueous suspensions for a long period of 3 months. Those studies suggest that block copolymers possess

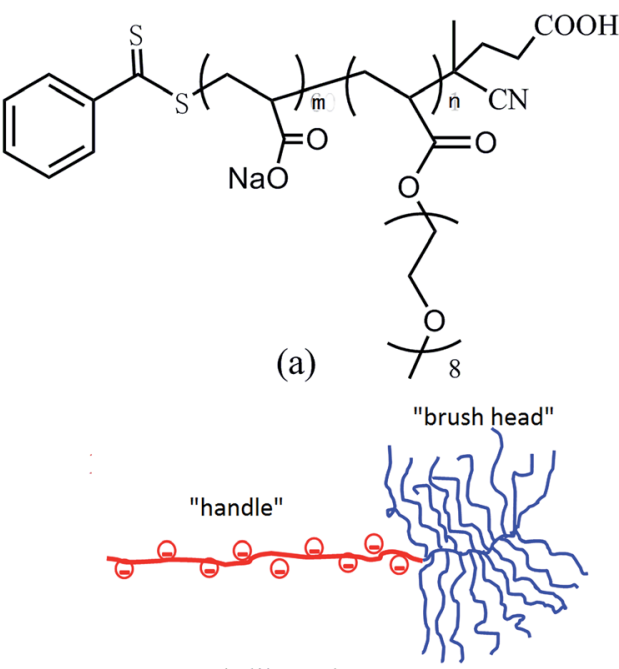

Brush-like polymer

(b)

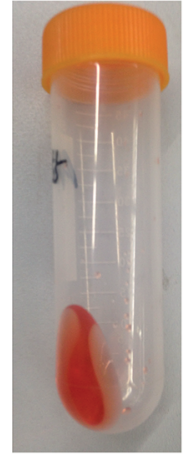

(c)
Fig. 1 Chemical structure (a), schematic illustration (b), and appearance (c) of the studied brush-like block copolymer. 
promising potential for a remarkable improvement of the stability of GO aqueous suspensions.

In this paper, a brush-like block copolymer, which has a "handle" made of polycarboxylate and a "brush-head" made of homo-polyoxyethylene, was synthesized via RAFT polymerization. Its chemical structure and polymer architecture are shown in Fig. 1a and b. The brush-like block copolymer was first applied as a dispersant to get stable GO aqueous suspensions. For the purpose of comparison, the dispersing performance of a commercial random copolymer polymer comprised of similar chemical units was also examined for the aqueous dispersion of GO.

\section{Experimental}

\subsection{Materials}

Oligo(ethylene glycol)methyl ether acrylate (OEGA, numberaverage molecular weight, $M_{\mathrm{n}}=480 \mathrm{~g} \mathrm{~mol}{ }^{-1}$ ), 4-cyano-4(phenylcarbonothioylthio)pentanoic acid (CPA) and 4,4'azobis(4-cyanovaleric acid) (ACVA) were purchased from Aldrich. $\mathrm{NaHCO}_{3}$, acrylic acid (AA), acetic acid and sodium acetate were purchased from Shanghai Chemical Reagent Co. Ltd. Exfoliated GO platelets (purity $>99 \%$, single layer ratio of $99 \%$, and average aspect ratio of 1600) were purchased from Nanjing XFnano (China) and used as received. The comparative random copolymer sample was purchased from Sobute Material Co. Ltd as the control, and was analyzed by proton nuclear

Table 1 Molecular weight and polydispersity index of the prepared brush-like block copolymer and the comparative random copolymer sample

\begin{tabular}{llll}
\hline & $M_{\mathrm{n}}$ & $M_{\mathrm{w}}$ & PDI \\
\hline Brush-like block copolymer & 13931 & 19760 & 1.42 \\
Purchased random & 9730 & 23150 & 2.38
\end{tabular}

magnetic resonance $\left({ }^{1} \mathrm{H}\right.$ NMR) spectroscopy and aqueous size exclusion chromatography. The ${ }^{1} \mathrm{H}$ NMR spectrum and molecular weight characteristics are given in Fig. 3 and Table 1 respectively.

\subsection{Synthesis of PAA- $b$-POEGA brush-like block copolymer}

The PAA- $b$-POEGA brush-like block copolymer was preparation via RAFT polymerization following a similar protocol as described in our previous work. ${ }^{22}$ In a typical experiment, the procedure was as follows. OEGA was added to $1 \mathrm{~mol} \mathrm{~L}^{-1}$ acetate buffer containing CPA and ACVA; the molar ratio among OEGA, CPA and ACVA was $50: 1: 0.1$, and the concentration of OEGA was $15 \%$ in weight. The solution was then purged with nitrogen for $30 \mathrm{~min}$ prior to the reaction. Polymerization was carried out under stirring at $70{ }^{\circ} \mathrm{C}$ maintained by a thermostat oil bath for $10 \mathrm{~h}$, and was quenched by immersion of the flask in ice water. The solution was then dialyzed against water, followed by reduced pressure distillation and freeze drying to obtain the intermediate product, which was called POEGA-RAFT. Next, POEGA-RAFT, AA and ACVA were added into $0.01 \mathrm{~mol} \mathrm{~L}^{-1}$ $\mathrm{NaHCO}_{3}$ aqueous solution with the molar ratio among AA, POEGA-RAFT and ACVA as $60: 1: 0.1$ and a POEGA-RAFT concentration of $15 \%$ in weight. The solution was deoxygenate with nitrogen for $30 \mathrm{~min}$, and was placed in a thermostated oil bath at $70{ }^{\circ} \mathrm{C}$ under stirring. After $8 \mathrm{~h}$, the polymerization was quenched by immersion of the flask in ice water. The mixture was dialyzed against water followed by freeze drying to obtain the final brush-like block copolymer product (as seen in Fig. 1c). Its ${ }^{1} \mathrm{H}$ NMR spectrum and molecular weight characteristics are given in Fig. 2 and Table 1, respectively, in comparison with the results for the random copolymer control sample.

\subsection{Characterization}

2.3.1 Zeta potential and size distribution of GO sheets. Zeta potential of GO aqueous suspensions was tested in $0.01 \mathrm{~mol} \mathrm{~L}^{-1} \mathrm{KCl}$ solution as background. GO aqueous

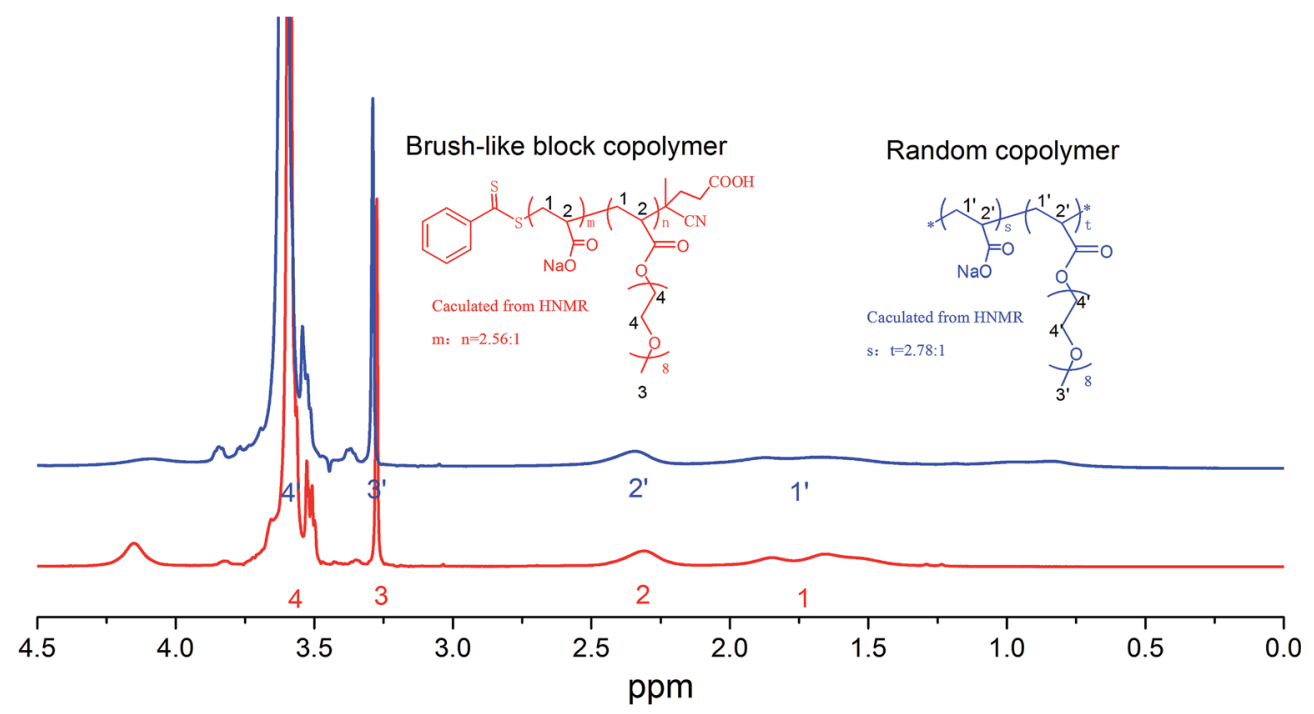

Fig. $2{ }^{1} \mathrm{H}$ NMR spectra of the prepared brush-like block copolymer and the comparative random copolymer sample. 
suspension with concentration $0.1 \mathrm{~g} \mathrm{~L}^{-1}$ was prepared by adding 1 part of GO powders into 10000 parts of $0.01 \mathrm{~mol} \mathrm{~L}^{-1} \mathrm{KCl}$ solution, and the suspension was placed under ultrasound for 15 minutes. The polymeric dispersant was added with concentration ranging from 0.5 to $16 \mathrm{mg} \mathrm{L}^{-1}$. Zeta potential of $\mathrm{GO}$ suspension was determined at $20 \pm 2{ }^{\circ} \mathrm{C}$ using a colloidal dynamics electroacoustic-based Zeta Probe analyzer (USA). The same method of sample preparation was applied to the size distribution characterization. Dynamic light scattering (DLS) was used to determine the size distributions of GO sheets using a photon correlation spectrometer (Brookhaven BI-200SM, US).

2.3.2 Adsorption measurements. The amount of polymer adsorbed was determined by means of a total organic carbon (TOC) analyzer, Multi N/C3100 (Analytik Jena AG, Germany). Sample preparation was the same as that described in Section 2.3.1. The aqueous phase was separated by centrifuging at $13000 \mathrm{rpm}$ for 10 minutes by a centrifuge. The supernatant was immediately decanted and dilute with deionized water for TOC analysis. Difference in the concentration before and after the incubation with GO was assumed to correlate directly with the amount of polymer adsorption.

2.3.3 Rheology and stability of GO aqueous suspensions. GO aqueous suspensions with concentration of $100 \mathrm{~g} \mathrm{~L}^{-1}$ were prepared by adding 10 part of GO powders into 90 parts of ultrapure water. Polymeric dispersant with amount equal to 1.5 wt $\%$ of GO powders was added to the suspensions. The suspensions were then placed under ultrasound for 15 minutes. Viscosity of the suspensions was measured immediately using a NX-11 type rotary viscosimeter (China) equipped with coaxial cylinder in controlled shear rate mode at $20{ }^{\circ} \mathrm{C}$. For the stability test, the suspensions were placed in little glass bottles on a stable table for 30 days, and photographs were taken in 1, 3 and 7 days to evaluate the stability of the GO aqueous suspensions visually.

2.3.4 SEM and XRD measurements. GO aqueous suspensions (prepared as described in Section 2.3.1) with and without polymer dispersants were dried via freeze-drying to obtain the black powders. Then, scanning electron microscopy (SEM, FEI Quanta 250, USA) and X-ray diffraction (XRD, Brookhaven D8 ADVANCE, USA) measurements were conducted to characterize the surface morphology and the interlayer spacing of the GO sheets.

\section{Results and discussion}

\subsection{Polymer adsorption and zeta potential}

Adsorption of polymer molecules onto the surface of GO sheets is believed to be key for the dispersing abilities of polymeric dispersants. Therefore, let's focus on the adsorption data first. Fig. 3a shows the amount of polymer adsorption as a function of
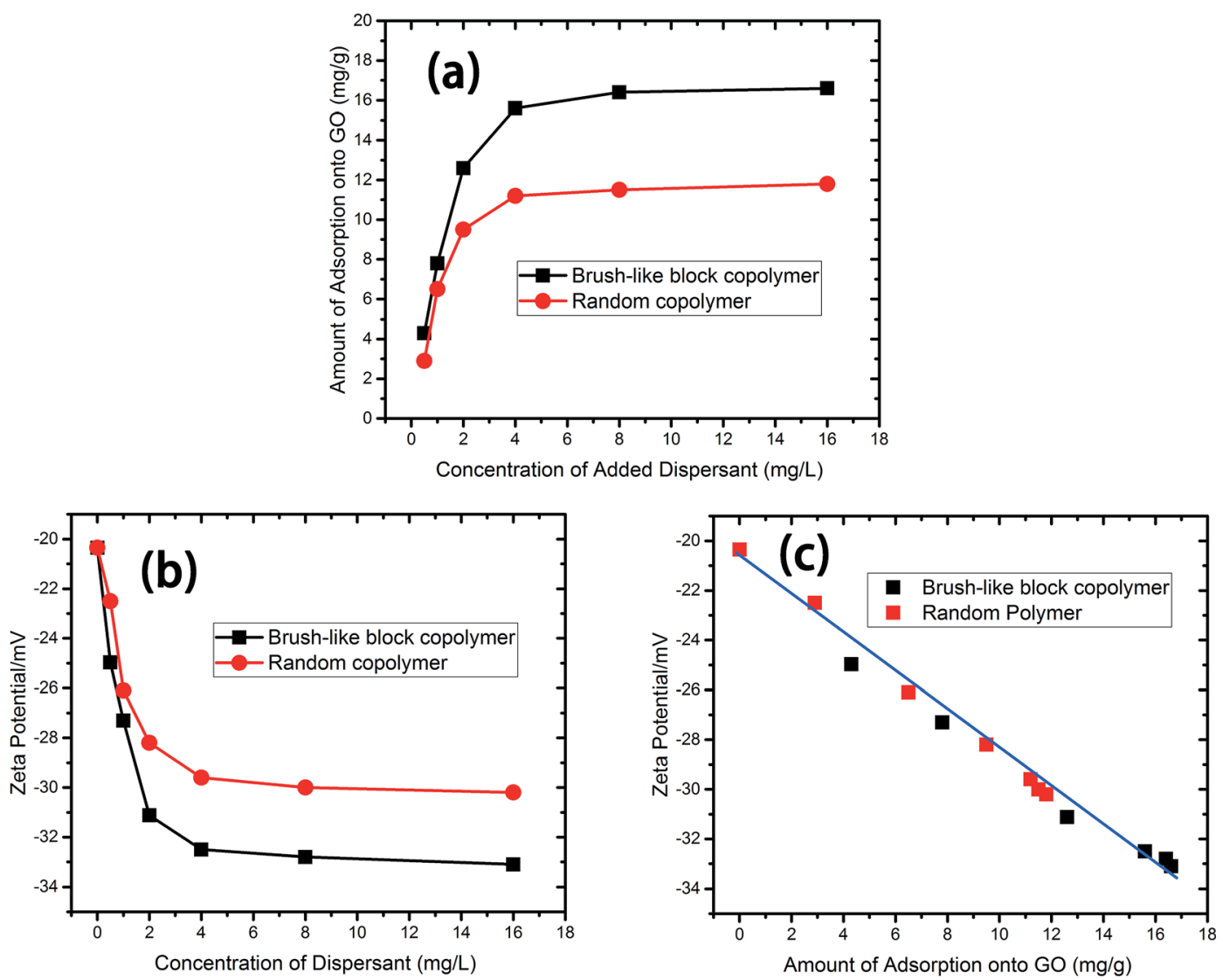

Fig. 3 Adsorption behavior (a) and variations in the zeta potential of GO suspensions as a function of the polymer concentration (b) and the amount of polymer adsorption (c) onto GO. 
the concentration of the added dispersants. It can be seen that, for both the brush-like block copolymer sample and the random copolymer control sample, the increase in the amount of adsorption with polymer concentration was more significant for low concentrations, and then gradually reached a plateau indicating surface saturation, when the solution concentration of the polymers was further increased, resembling a typical Langmuir-type adsorption isotherm. The difference in the saturated amount of adsorption also indicates a difference in the adsorbed conformations and the surface occupation area. At the same polymer concentration, the brush-like block copolymer had a larger amount of adsorption, which is probably due to a minimal steric hindrance caused by the neutral side chains and a higher charge density (more concentrated charges) along the "handle" block of the brush-like block copolymer than that of the random copolymer. In an aqueous salt solution, the surface of GO is negative charged with an electric double layer formed on the surface of GO. The charged polymers are adsorbed onto the surface of GO due to the electric double layer as well as entropic effects. For the brush-like block copolymer, the carboxyl groups in the brush-like copolymer are more exposed and have less shielding effects from the OEGA branches, whereas for the control sample, the carboxyl groups are sheltered by the polyoxyethylene chains, and polymer entropy prevents the approaching of the carboxyl groups along
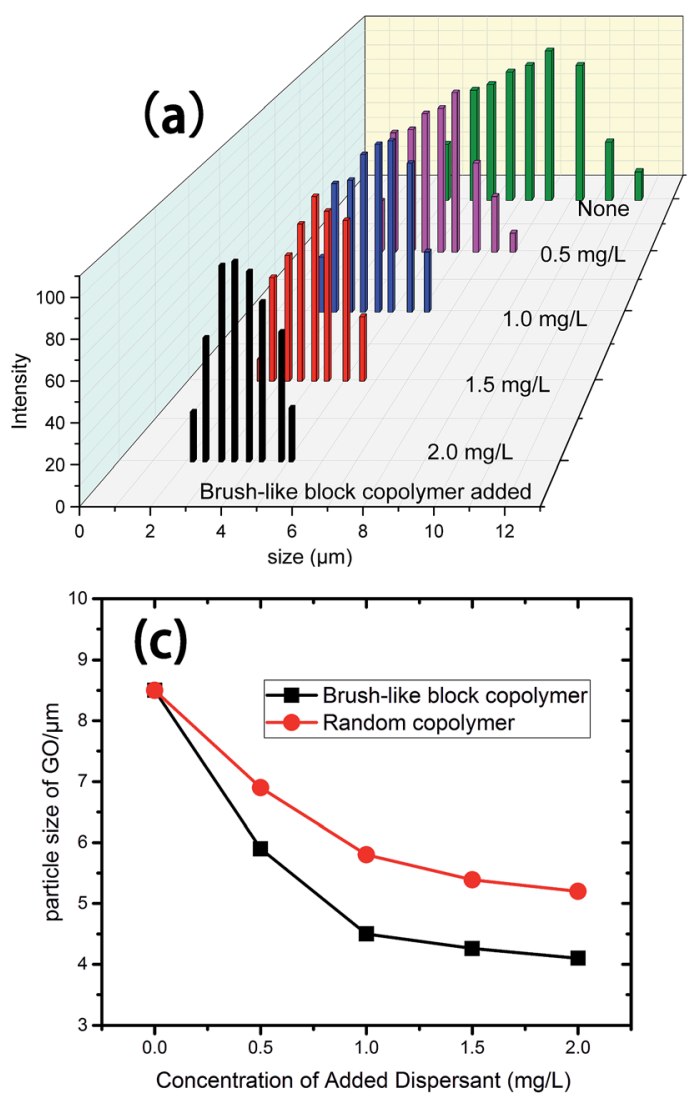

the polymer backbone to the surface of GO sheets. Thus, the brush-like block copolymer shows more adsorption.

GO suspensions with different content of dispersant have different values of zeta potential. Fig. $3 \mathrm{~b}$ presents the zetapotentials as a function of the concentration of added dispersant. The addition of polymers led to a decrease in the zeta potential, and with the increase of polymer concentration, the zeta-potentials became more negative for both systems and eventually reached their respective plateau values. It is seen that the brush-like block copolymer led to a more negative zeta potential of the surfaces of GO sheets than the random copolymer control, which may be expected from the higher adsorption amount (see Fig. 3a) of the brush-like block copolymer. Fig. $3 c$ presents the zeta-potentials $v s$. the amount of polymer adsorption. It is seen that the zeta potential decreased nearly linearly with the amount of polymer adsorption. It is also interesting that the results for both types of polymers had no obvious difference in their correlation between zeta potential and the adsorbed amount. Fig. $3 \mathrm{c}$ gives evidence that zeta potential may be used to assess the amount of polymer adsorption on the surfaces of GO sheets.

\subsection{Particle size analysis}

The stability of GO suspensions relates closely to the particle sizes and the size distribution of the GO sheets. If no dispersant
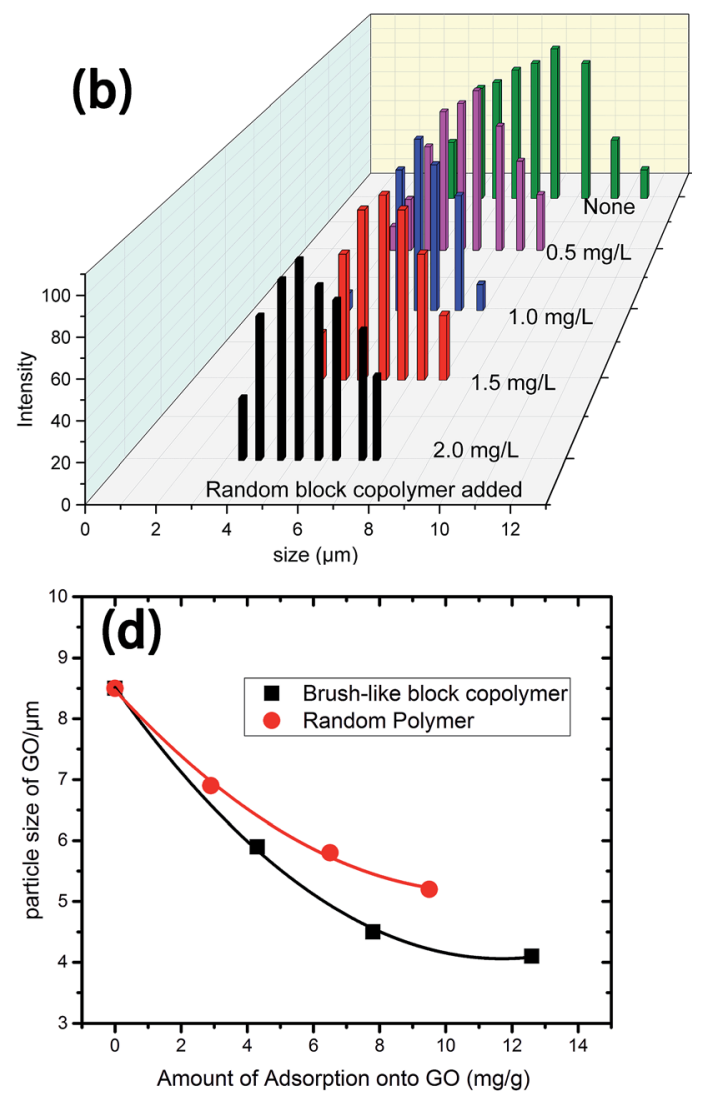

Fig. 4 Size distributions of GO suspensions with the prepared brush-like block copolymer (a) and the comparative random copolymer sample (b) at different polymer concentrations; (c) and (d) shows the average particle size of GO suspensions as a function of the polymer concentration (c) and the amount of polymer adsorption (d), respectively. 

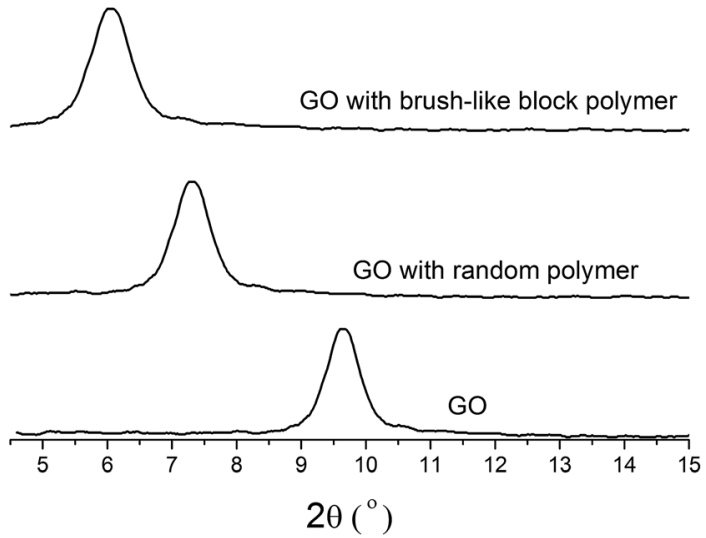

Fig. 5 Comparison of the small angle XRD patterns for GO suspensions with and without dispersant.

was added, the GO sheets intended to gather together to form larger aggregates. With the addition of dispersants, such aggregation processes could be prevented to some extent. As shown in Fig. 4a and b, the particle size distribution is narrowed towards smaller particle sizes with the addition of both types of polymeric dispersants. At the same polymer concentration, the brush-like block copolymers could prevent the aggregation more effective than the random copolymer control, as suggested by Fig. 4c, where the average particle size is shown as a function of added polymer concentration. When adding brush-like block copolymer as dispersant, the average size of GO sheets decreased from $8.5 \mu \mathrm{m}$ to $5.8 \mu \mathrm{m}$ at a polymer concentration of $0.5 \mathrm{mg} \mathrm{L}^{-1}$, and by increasing the polymer concentration, the average size of GO sheets decreased furthered to about $4.2 \mu \mathrm{m}$ at a polymer concentration of $2.0 \mathrm{mg} \mathrm{L}^{-1}$. The random polymer showed a similar effect, but the average size of GO sheets with random polymer as dispersant was larger than that with brushlike polymer as dispersant at the same polymer concentration. Fig. $4 \mathrm{~d}$ shows the average particle size versus the amount of

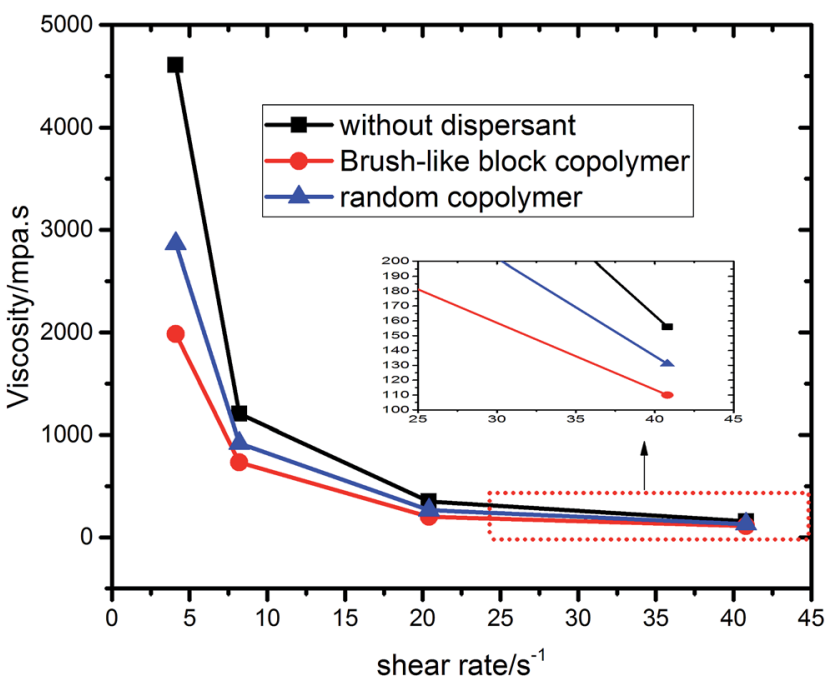

Fig. 6 Viscosity vs. shear rate for GO aqueous suspensions with and without dispersant. polymer adsorption, and it showed that more polymer adsorption correlated with a smaller average particle size, and at the same adsorbed amount, the brush-like block polymer sample resulted in a smaller average particle size, which indicates a better dispersing ability than the random copolymer control. The dispersing ability of polymer dispersant is believed to correlate with the electrostatic interactions and maybe more importantly, the steric hindrance effects introduced by the side chains. The brush-like block polymer and random polymer had no obvious difference in changing the surface zeta-potential of GO sheets (see Fig. 3c) at the same amount of adsorption, and therefore their difference in dispersing ability is probably caused by a difference in steric hindrance effects. Our interpretation is as follows. The brush-like block polymer provides a stronger steric hindrance because of the relatively larger "brush-head" made of homo-polyoxyethylene and the randomly distributed side chains of the control sample. To validate this hypothesis, Fig. 5 gave the small angle XRD patterns of GO sheets with and without dispersant. GO sheets without adding dispersant had a peak of $2 \theta=9.7^{\circ}$, corresponding to an interlayer spacing of $0.91 \mathrm{~nm}$. When using the brush-like block polymer and the random polymer control as dispersant, at a similar adsorption amount of $8 \mathrm{mg} \mathrm{g}^{-1}$, the peak shifted to $2 \theta$ $=6.5^{\circ}$ and $7.6^{\circ}$, corresponding to an interlayer spacing of $1.36 \mathrm{~nm}$ and $1.15 \mathrm{~nm}$, respectively. This result gave evidence to the stronger steric hindrance effect of the brush-like block

\section{Initial 1day 3days 7days}

(a)
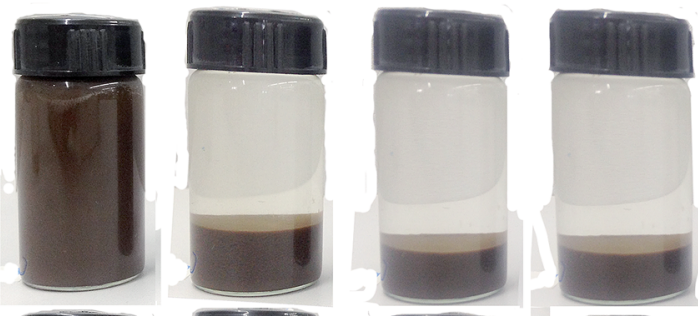

(b)
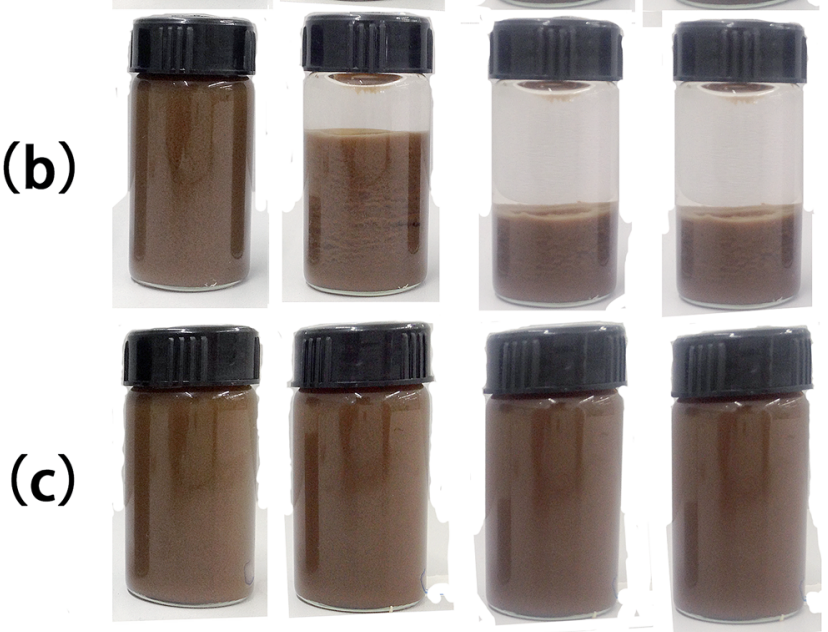

Fig. 7 Photographs of $\mathrm{GO}$ aqueous suspensions at different storage time for samples without dispersant (a) and with the comparative random copolymer (b) or the prepared brush-like copolymer (c) as dispersant. 

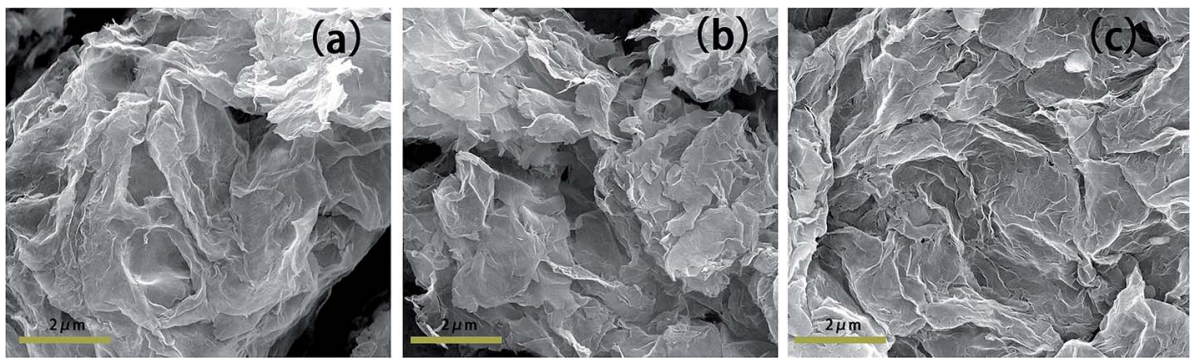

Fig. 8 SEM images of GO sheets for samples, prepared without dispersant (a) and with the comparative random copolymer (b) or with the brushlike copolymer (c) as dispersant, after freeze-drying.

polymer than that of the random copolymer, and it is believed that this difference in steric hindrance effect is related to their difference in adsorption conformations, which is then related to their architectures.

\subsection{Shear viscosity}

Rheological data can also be used to assess the quality of dispersion of GO aqueous suspension. Here, the shear viscosity of GO aqueous suspensions with and without dispersant was measured at a concentration of GO suspension as high as $100 \mathrm{~g}$ $\mathrm{L}^{-1}$. The dosage of the dispersant was fixed at $1.5 \%$ of the weight of GO. The shear rate was varied to study the shear-thinning or pseudoplasticity of GO suspensions. As shown in Fig. 6, the GO suspension without dispersant had a viscosity value as high as 4.6 $\mathrm{Pa} \mathrm{s}$ at a low shear rate of about $5 \mathrm{~s}^{-1}$; with the addition of polymers, the shear viscosity of GO aqueous suspensions decreased to $\sim 2.0$ and $\sim 2.8$ Pa s, respectively, for the brush-like block copolymer and the random copolymer as dispersant. With the increase of shear rate, the shear viscosity further decreased, suggesting a shear-thinning behavior. At a high shear rate of about $40 \mathrm{~s}^{-1}$, all samples had a similarly low shear viscosity of about $150 \mathrm{mPa}$ s. However, the GO suspension with brush-like block copolymer still had the least viscosity of $\sim 110 \mathrm{mPa} \mathrm{s}$ as shown by the zoomed-in view in Fig. 6 . Thus, it is fair to conclude that the brush-like block copolymer performs well on decreasing the shear viscosity of GO aqueous suspensions.

\subsection{Stability of GO aqueous suspensions}

To monitor more directly the stability of GO aqueous suspensions, $20 \mathrm{~mL}$ of $\mathrm{GO}$ aqueous suspension with and without dispersant were stored in little glass bottles, and photographs were taken at 0 time and after 1 day, 3 days and 7 days to record the extent of precipitation of the suspensions. The results are shown in Fig. 7. Clearly, the GO particles in aqueous suspension without dispersant precipitated drastically to the bottom within 1 day or so; using the random copolymer as dispersant, the GO aqueous suspension showed obvious precipitation of particles within 3 days; however, with the brush-like block copolymer as dispersant, the GO aqueous suspension stayed homogeneous (at least macroscopically) over 7 days, with almost no apparent change with its initial appearance at time 0 .

Fig. 8 showed the surface morphology of GO sheets without and with polymer dispersants after freeze-drying. Without adding any dispersant, GO sheets aggregated into clusters (Fig. 8a). By adding polymeric dispersant (Fig. 8b and c), the aggregates of GO sheets were noticeably smaller, and thin-layer structures were observed, which were believed to be helpful for the stability of GO aqueous suspensions. Moreover, it seemed that such thin-layer structure were more abundant in Fig. 8c when the brush-like block copolymer was used as the polymeric dispersant, also suggesting a better dispersing ability than the random copolymer sample.

\section{Conclusions}

PAA-b-POEGA brush-like block copolymer was synthesized via RAFT polymerization, and its dispersing ability for GO sheets in aqueous suspensions were examined using a commercial random copolymer with similar chemical units as the control group. The brush-like block copolymer could adsorb onto the surface of GO, and the amount of polymer adsorption correlated well with the decrease in the zeta potential of the GO sheets. The brush-like block copolymer was found to have effective dispersing ability for GO sheets in aqueous suspensions. Using the brush-like block copolymer as dispersant, GO sheets possessed smaller average particle size, narrower size distribution and lower shear viscosity than the control groups. The GO aqueous suspensions were stable at a GO concentration as high as $100 \mathrm{~g} \mathrm{~L}^{-1}$ for 7 days using the brush-like block copolymer as dispersant, which cannot be accomplished by using traditional random copolymer as dispersant at the same added amount.

\section{Acknowledgements}

This work is supported by the National Natural Science Foundation of China (No. 51408271, 51272100 and 51273073).

\section{References}

1 K. S. Novoselov, A. K. Geim, S. V. Morozov, D. Jiang, Y. Zhang, S. V. Dubonos, I. V. Grigorieva and A. A. Firsov, Electric field effect in atomically thin carbon films, Science, 2004, 306, 666-669.

2 V. C. Tung, M. J. Allen, Y. Yang and R. B. Kaner, Highthroughput solution processing of large-scale graphene, Nat. Nanotechnol., 2009, 4, 25-29. 
3 Y. Zhao, L. Song, Z. Zhang and L. Qu, Stimulus-responsive graphene systems towards actuator applications, Energy Environ. Sci., 2003, 6, 3520-3536.

4 E. Wang, M. S. Desai and S. W. Lee, Light-controlled graphene-elastin composite hydrogel actuators, Nano Lett., 2016, 13, 2826-2830.

5 N. Mahmood, C. Zhang, H. Yin and Y. Hou, Graphene-based nanocomposites for energy storage and conversion in lithium batteries, supercapacitors and fuel cells, J. Mater. Chem. A, 2014, 2, 15-32.

6 V. Tozzini and V. Pellegrini, Prospects for hydrogen storage in graphene, Phys. Chem. Chem. Phys., 2013, 15, 80-89.

$7 \mathrm{H}$. Chang and H. Wu, Graphene-based nanocomposites: preparation, functionalization, and energy and environmental applications, Energy Environ. Sci., 2013, 6, 3483-3507.

8 A. Asthana, T. Maitra, R. Buchel, M. K. Tiwari and D. Poulikakos, Multifunctional Superhydrophobic Polymer/ Carbon Nanocomposites: Graphene, Carbon Nanotubes, or Carbon Black, ACS Appl. Mater. Interfaces, 2014, 6, 88598867.

9 M. Wang, Y. Niu, J. Zhou, H. Wen, Z. Y. Zhang, D. Luo, D. L. Gao, J. Yang, D. H. Liang and Y. Li, The dispersion and aggregation of GO in aqueous media, Nanoscale, 2016, 8, 14587-14592.

10 H. T. Larijani, M. D. Ganji and M. Jahanshahi, Trends of amino acid adsorption onto graphene and GO surfaces: a dispersion corrected DFT study, $R S C A d v$., 2015, 5, 92843-92857.

11 M. Lotya, P. J. King, U. Khan, S. De and J. N. Coleman, HighConcentration, Surfactant-Stabilized Graphene Dispersions, ACS Nano, 2010, 4, 3155-3162.

12 M. S. Zhu, M. S. Zhu, Z. Li, B. Xiao, Y. T. Lu, Y. K. Du, P. Yang and X. M. Wang, Surfactant Assistance in Improvement of Photocatalytic Hydrogen Production with the Porphyrin Noncovalently Functionalized Graphene Nanocomposite, ACS Appl. Mater. Interfaces, 2013, 5, 1732-1740.

13 A. S. Wajid, S. Das, F. Irin, H. S. T. Ahmed, J. L. Shelburne, D. Parviz, R. J. Fullerton, A. F. Jankowski, R. C. Hedden and M. J. Green, Polymer-stabilized graphene dispersions at high concentrations in organic solvents for composite production, Carbon, 2012, 50, 526-534.

14 J. W. T. Seo, A. A. Green, A. L. Antaris and M. C. Hersam, High-Concentration Aqueous Dispersions of Graphene Using Nonionic, Biocompatible Block Copolymers, J. Phys. Chem. Lett., 2011, 2, 1004-1008.

15 L. Guardia, M. J. Fernández-Merino, J. I. Paredes, P. SolísFernández, S. Villar-Rodil, A. Martínez-Alonso and J. M. D. Tascón, High-throughput production of pristine graphene in an aqueous dispersion assisted by non-ionic surfactants, Carbon, 2011, 49, 1653-1662.

16 R. Feng, W. Zhou, G. Guan, C. Li, D. Zhang, Y. Xiao, L. Zheng and W. Zhu, Surface decoration of graphene by grafting polymerization using GO as the initiator, J. Mater. Chem., 2012, 22, 3982-3989.

17 Z. S. Tian, C. X. Xu, J. T. Li, G. Y. Zhu, Z. L. Shi and Y. Lin, Self-Assembled Free-Standing GO Fibers, ACS Appl. Mater. Interfaces, 2013, 5, 1489-1493.

18 S. Das, F. Irin, H. S. Tanvir Ahmed, A. B. Cortinas, A. S. Wajid, D. Parivz, A. F. Jankowski, M. Kato and M. J. Green, Non-covalent functionalization of pristine fewlayer graphene using triphenylene derivatives for conductive poly(vinyl alcohol) composites, Polymer, 2012, 53, 2485-2494.

19 Y. B. Yan, L. H. Piao, S. H. Kim, W. Lia and H. Zhou, Effect of Pluronic block copolymers on aqueous dispersions of GO, RSC Adv., 2015, 5, 40199-40204.

20 M. L. Hyang, S. Perumal and I. W. Cheong, Amphiphilic Fluorinated Block Copolymer Synthesized by RAFT Polymerization for Graphene Dispersions, Polymers, 2016, 8, 101-112.

21 S. Perumal, K. T. Park, M. L. Hyang and I. W. Cheong, PVP- $b$ PEO block copolymers for stable aqueous and ethanolic graphene dispersions, J. Colloid Interface Sci., 2016, 464, 25-35.

22 C. Yu, Q. Chen, A. Wang, X. Zhou, S. Wu and Q. Ran, Improved dispersibility of multi-wall carbon nanotubes with reversible addition-fragmentation chain transfer polymer modification, Polym. Int., 2015, 64, 1219-1224. 